\title{
Solitary Fibrous Tumor in Buccal Cheek Mucosa
}

\author{
Chung-Min Yoon ${ }^{1}$, \\ Jeong-Min $\mathrm{Cho}^{1}$, \\ Kwang-Ryeol Lim', \\ Seok-Kwun Kim', \\ Su-Jin $\mathrm{Kim}^{2}$, \\ Keun-Cheol Lee ${ }^{1}$
}

Departments of ${ }^{1}$ Plastic and Reconstructive Surgery and ${ }^{2}$ Pathology, Dong-A University School of Medicine, Busan, Korea

No potential conflict of interest relevant to this article was reported.

\begin{abstract}
A solitary fibrous tumor is a relatively uncommon neoplasm that usually occurs in the pleura but occurs extremely rarely in the oral cavity. Reported herein is a rare case of a solitary fibrous tumor in the buccal cheek mucosa. A 50-year-old man visited the authors' hospital due to a buccal cheek mass whose size had increased. Excisional biopsy was done under local anesthesia. After the excisional biopsy, the patient was diagnosed to have a solitary fibrous tumor. In immunohistochemistry, the patient's solitary fibrous tumor was characterized by the expression of CD34 and CD99 on the neoplastic cells, and negativity for Bcl-2 and S-100. No recurrence or complication occurred for a period of 5 years. The growth of a primary solitary fibrous tumor in the buccal cheek mucosa is extremely rare and has been rarely reported in the South Korean medical literature. A solitary fibrous tumor must be distinguished from other spindle cell tumors. Presented herein is a case of primary solitary fibrous tumor in the buccal cheek mucosa. The relevant literature is briefly reviewed.
\end{abstract}

Keywords: Solitary fibrous tumor / Buccal mucosa / Cheek

\section{INTRODUCTION}

Solitary fibrous tumor is a tumor of mesenchymal origin that was first described by Klemperer and Rabin [1] and is known to occur mainly in the pleura. It has also been reported to develop in various other locations, however, such as the abdominal cavity, upper respiratory tract, and soft tissue, but its occurrence in the oral cavity is extremely rare, and there have been few reported cases of such in South Korea. Solitary fibrous tumor can be treated by surgical excision, and immunofluorescence staining is employed for differential diagnosis with factors like CD34 and CD99. In the authors' Department, there has been one case of diagnosis with solitary fibrous tumor after total resection and histopathologic examination for a buccal cheek mass. Such rare case is reported herein.

\section{Correspondence: Keun-Cheol Lee}

Department of Plastic and Reconstructive Surgery, Dong-A University school of

Medicine. 26 Daesingongwon-ro, Seo-gu, Busan 49201, Korea

E-mail: pokdungi@dau.ac.kr

*This study was supported by research funds from Dong-A University.

Received September 6, 2017 / Revised September 20, 2017 / Accepted September 20, 2017

\section{CASE REPROT}

The patient was a 50-year-old man with a touchable mass in the buccal mucosa of the right cheek who visited the Plastic Surgery Outpatient Department of Dong-A University Hospital as the size of the mass had become larger. The patient did not have any unusual condition, except for histories of open reduction and internal fixation for LeFort I \& II fracture due to falling. The patient thought that the mass developed after he incurred such injury. There was no specific finding at the visual inspection, and a softtissue lump that felt slightly hard was touched upon palpation. In the mouth, an approximately $2 \mathrm{~cm}$ mass covered by normal mucosal tissues was uplifted (Fig. 1A). Under local anesthesia, excisional biopsy was performed, and after the complete removal of the lesion, the surgical site was sutured. The tissue that had been removed had clear boundaries and was covered with a fibrous capsule. The mass was well demarcated, oval, and $1.5 \mathrm{~cm}$ in size, showing a diffuse fibrotic appearance. The tissue that had been obtained through the surgery was fixed in $10 \%$ formalin, and hematoxylin \& eosin (H\&E) staining and immunofluorescence staining were done. In the H\&E staining, the mass was found to 
have been composed of abundant collagenous stroma admixed with bland-looking tumor cells (Fig. 2). In the immunofluorescence staining, positive responses to CD34 and CD99 (Fig. 3) and negative responses to Bcl-2, S-100, and smooth muscle actin (SMA) were shown. There was no finding of inflammation or re-
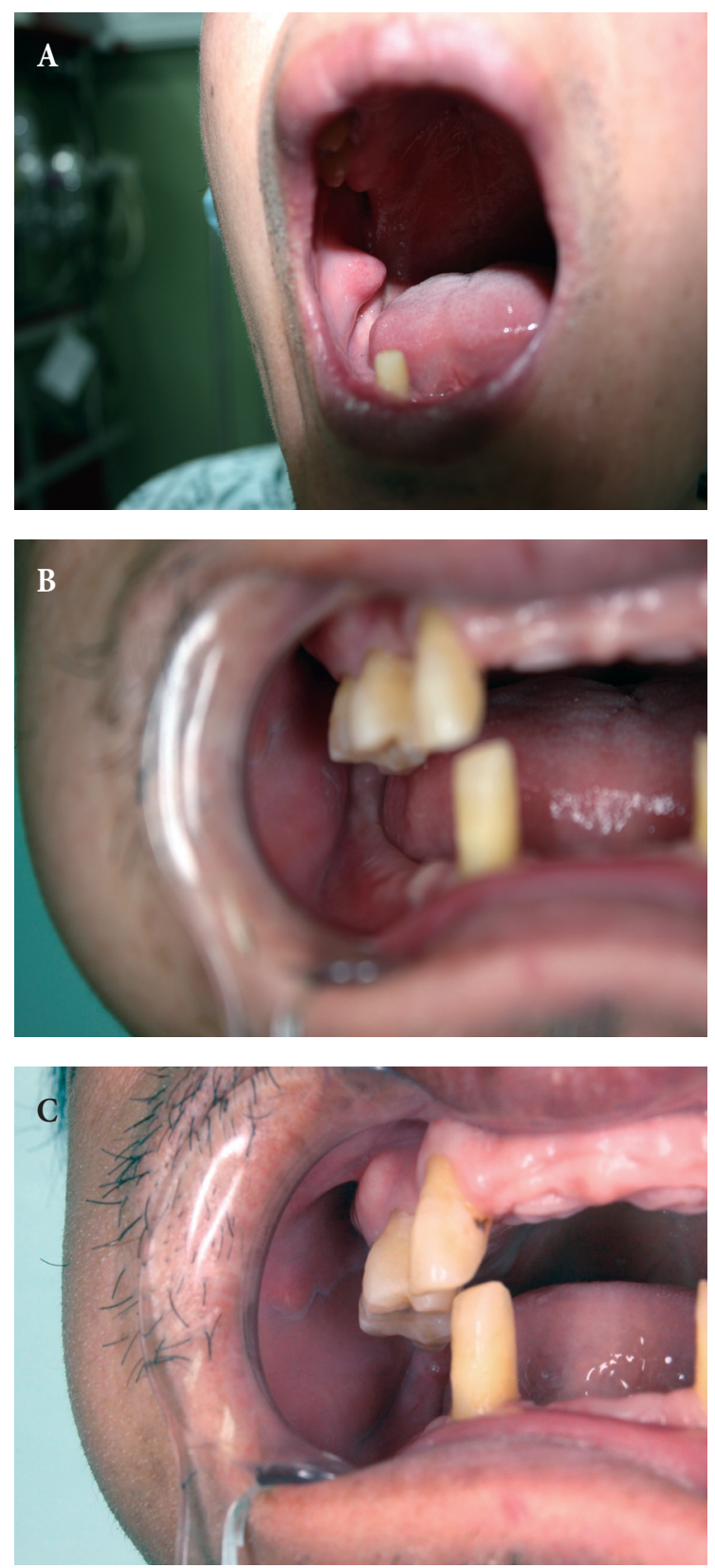

Fig. 1. (A) Preoperative view. (B) Postoperative finding in 3 months after the surgery. (C) Postoperative finding in 5 years after the surgery. There was no recurrence or complications.

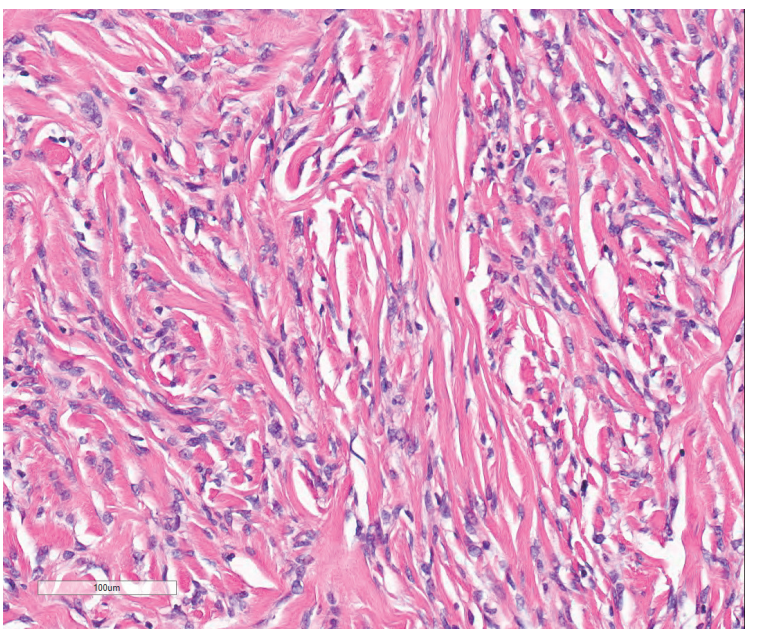

Fig. 2. The mass is composed of abundant collagenous stroma admixed with bland looking tumor cells $(\mathrm{H} \& \mathrm{E}, \times 200)$.
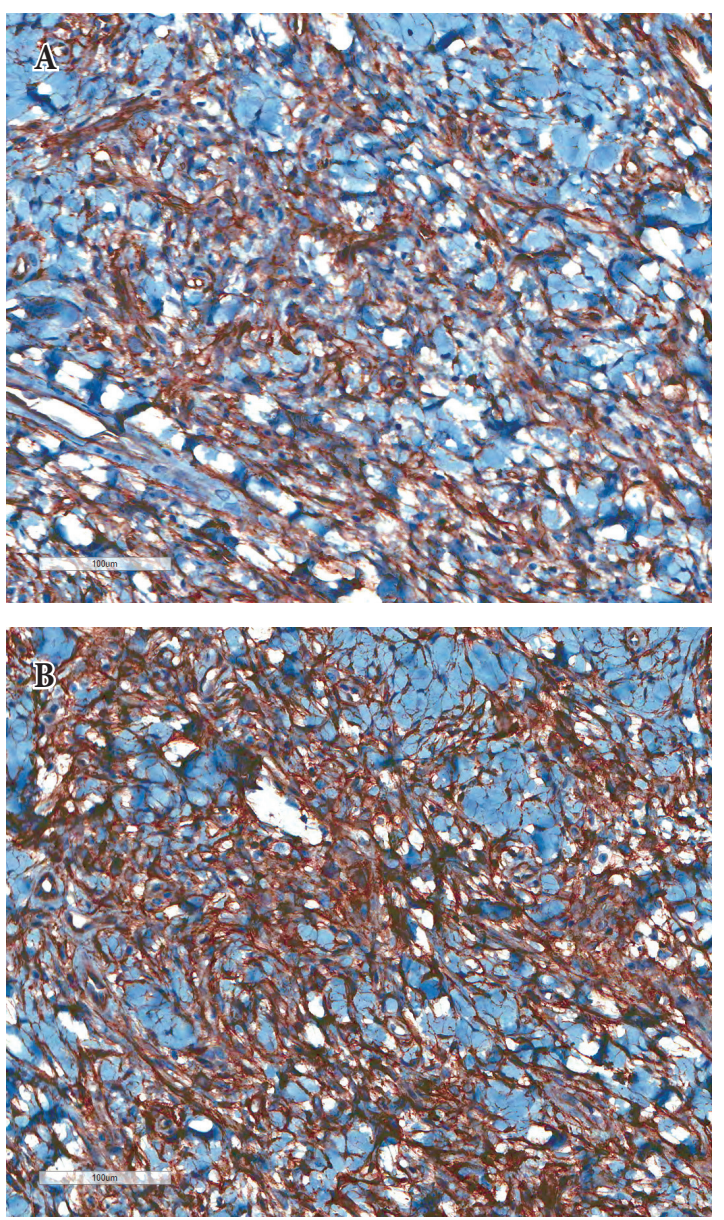

Fig. 3. (A, B) The tumor cells are immunoreactive for CD34 \& CD99 $(\times 200)$, which is consistent feature with solitary fibrous tumor. 
currence 3 months and 5 years after the surgery (Fig. 1B, C).

\section{DISCUSSION}

Solitary fibrous tumor is a tumor of mesenchymal origin and is known to occur mainly in the pleura but also occurs in the peritoneum, pericardium, and mediastinum. It was recently reported to have occurred in the epithelial organs, such as the thyroid, paranasal sinus, orbital soft tissue, lacrimal gland, and skin [2-4]. In the 153 cases of solitary fibrous tumor that developed in the head and neck that were examined by Cox et al. [5], 26.1\% developed in the buccal mucosa, $9.2 \%$ in the nasal cavity, $7.8 \%$ in the pharynx, $7.2 \%$ in the tongue, and $6.5 \%$ in the orbit. Solitary fibrous tumors in the head and neck are relatively rare compared to other sites. As there are not many cases, there are no definite diagnosis criteria, but they can be diagnosed via computed tomography (CT), magnetic resonance imaging, and positron emission tomography-CT. Neurofibroma, schwannoma, leiomyoma, and hemangiopericytoma are the lesions that need to be differentiated. For differential diagnosis, $\mathrm{H} \& \mathrm{E}$ staining and immunofluorescence staining are used.

Through H\&E staining, the cell array pattern in the solitary fibrous tumor can be observed. The cell array pattern is characterized by various findings, including the arrangement of fusiform cells with a histologically relatively regular shape and size but without a particular form, a swirling growth style, the appearance of enlarged vessels observed in the mesothelioma of the blood vessels, fibrosarcoma-shaped growth, and a substrate with a low cell density and with enriched acidophil fibers [6]. In immunofluorescence staining, a positive response to CD34 is shown, and mast cells are shown to be abundant in the solitary fibrous tumor because such tumor originates from a CD34-positive pluripotential cell. CD99 is also known to be helpful at the time of diagnosis because the solitary fibrous tumor shows positive responses in varying degrees. The CD34 antigen is a single-chain glycoprotein inside and outside the membrane of $115 \mathrm{kDa}$ that is selectively expressed in human hematopoietic progenitor cells and endothelial cells. It is also found in the mesenchymal and interstitial cells and in various types of mesenchymal tumors [7].
In the case reported herein, positive responses to CD34 and CD99 and negative responses to $\mathrm{Bcl}-2, \mathrm{~S}-100$, and SMA were shown. Neurofibroma and schwannoma may be positive to CD34 and $\mathrm{Bcl}-2$ but is negative to $\mathrm{S}-100$; thus, they can be easily differentiated. Leiomyoma is positive to SMA but is negative to CD34 and Bcl-2; thus, it can be easily differentiated. It should be noted that CD34 is expressed not only in solitary fibrous tumor but in many other types of tumor cells, such as the dermatofibrosarcoma protuberans, spindle cell lipoma, and hemangiopericytoma. As a result, it is difficult to use CD34 as a specific marker of solitary fibrous tumor. Moreover, Vimentin, Bcl-2, CD99, and Factor XIIIa are also expressed in other tumor cells. Therefore, there is no solitary-fibrous-tumor-specific marker to date, and it is necessary to make a diagnosis by comprehensively considering the histologic findings and expressions of various markers [8].

As the solitary fibrous tumor usually grows slowly and without pain, the patient is often not aware of its presence until it has grown to a certain size. In the case of a solitary fibrous tumor that developed in the mouth, in most cases, the patients visit the hospital with a major complaint of dysarthria or masticatory disorder [9].

Solitary fibrous tumor is treated by surgical total resection. Most tumors are benign, and the prognosis is known to be good. Most solitary fibrous tumors show a single type of tumor cell proliferation findings, but malignant findings may also be shown in a few cases. If cell polymorphism, high cell fidelity, a number of division patterns (4 or more in high-magnification findings), and necrosis of the tumor cells are shown, the possibility of malignant degeneration should be considered, and association with a high recurrence rate and distant metastasis is indicated [10]. In addition, more malignant cases of solitary fibrous tumor in the extremities have been reported in another study [11]. If malignancy is suspected based on the biopsy finding, a more aggressive treatment should be considered, such as wide excision. The case reported herein was judged as a benign tumor, and recurrence was not observed 5 years later, but as it may recur, long-term follow-up is necessary [9].

Reported herein is a resolved case of a 50-year-old male patient with a facial fracture history who visited the hospital with a major 
complaint of a subcutaneous mass that had developed in the buccal mucosa on his right cheek. After the performance of total resection under the suspicion of the presence of a mucosal polyp or fibroid that had developed due to the patient's previous injury, solitary fibrous tumor was diagnosed as a result of the biopsy that was conducted. As the development of solitary fibrous tumor in the buccal mucosa is extremely rare in the South Korean medical literature, the case was reported along with a literature review.

\section{PATIENT CONSENT}

The patient provided written informed consent for the publication and the use of their images.

\section{REFERENCES}

1. Klemperer P, Rabin CB. Primary neoplasm of the pleura: a report of five cases. Arch Pathol 1931;11:385-412.

2. Lee NH, Ahn BC, Moon WS. A case of solitary fibrous tumor of lacrimal gland. J Korean Ophthalmol Soc 2000;41:234-8.
3. Morales-Cadena M, Zubiaur FM, Alvarez R, Madrigal J, Zarate-Osorno A. Solitary fibrous tumor of the nasal cavity and paranasal sinuses. Otolaryngol Head Neck Surg 2006;135:980-2.

4. Kang TW, Kim HJ, Kim YC, Kim SC. A case of solitary fibrous tumor that developed on the scalp. Korean J Dermatol 2009;47:615-7.

5. Cox DP, Daniels T, Jordan RC. Solitary fibrous tumor of the head and neck. Oral Surg Oral Med Oral Pathol Oral Radiol Endod 2010;110:79-84.

6. Moon WS, Lee KM. Solitary fibrous tumor clinico: pathological analysis of 11 cases. J Korean Cancer Assoc 1999;31:847-54.

7. Westra WH, Gerald WL, Rosai J. Solitary fibrous tumor: consistent CD34 immunoreactivity and occurrence in the orbit. Am J Surg Pathol 1994;18:992-8.

8. Oh YJ, Lew BL, Sim WY. Primary cutaneous solitary fibrous tumor. Korean J Dermatol 2011;49:1093-7.

9. Migita M, Yoshino M, Kobayashi D, Shiomi S, Enatsu K, Shigematsu S, et al. A large solitary fibrous tumor of the tongue. J Oral Maxillofac Surg 2012;70:871-4.

10. Shnayder Y, Greenfield BJ, Oweity T, DeLacure MD. Malignant solitary fibrous tumor of the tongue. Am J Otolaryngol 2003;24:246-9.

11. Anders JO, Aurich M, Lang T, Wagner A. Solitary fibrous tumor in the thigh: review of the literature. J Cancer Res Clin Oncol 2006;132:69-75. 